\section{Elenco de medicamentos do Programa Farmácia Popular do Brasil e a Política de Nacional Assistência Farmacêutica}

\author{
The list of drugs in the Popular Pharmacy Program \\ and the Brazilian National Pharmaceutical \\ Care Policy
}

\author{
Lista de medicamentos del Programa Farmacia \\ Popular de Brasil y la Política Brasileña \\ de Asistencia Farmacéutica
}

Sueli Miyuki Yamauti 1,2 Silvio Barberato-Filho 2 Luciane Cruz Lopes 2

\footnotetext{
${ }^{1}$ Hospital Universitário, Universidade Federal de São Paulo, São Paulo, Brasil. 2 Universidade de Sorocaba, Sorocaba, Brasil.

Correspondência S. M. Yamauti Rua Castro Alves 279, São Paulo, SP 01532-001, Brasil. sueli.gmo@epm.br
}

\begin{abstract}
This study aimed to analyze the list of drugs in the Popular Pharmacy Program in Brazil (PF$P B)$ in relation to the country's pharmaceutical care policy. The list of drugs in the PFPB was compared to the Brazilian and international reference lists of essential medicines, the components of pharmaceutical care in Brazilian Unified National Health System (SUS), and drug production by the country's government pharmaceutical laboratories. The PFPB list includes 119 drugs, of which $19.3 \%$ and $47.1 \%$ were not selected on the Brazilian and international reference lists, respectively; $16.8 \%$ are not used in primary care, and $40.3 \%$ are not produced by the country's government laboratories. A revision of the PFPB list based on the essential medicines concept (World Health Organization), alignment of pharmaceutical care policies, and production by government laboratories are essential to improve quality of health care, management, training of prescribers, and information for the population.
\end{abstract}

Essential Drugs; Pharmaceutical Services; National Health Programs; Primary Health Care

\section{Resumo}

O objetivo deste trabalho foi analisar o elenco de medicamentos do Programa Farmácia Popular do Brasil (PFPB) frente à política de assistência farmacêutica vigente no país. O elenco de medicamentos do PFPB foi comparado com listas de referência de medicamentos essenciais (nacional e internacional), com os componentes da assistência farmacêutica no Sistema Único de Saúde (SUS) e com a produção pública de medicamentos nos Laboratórios Farmacêuticos Oficiais do Brasil (LFOB). O PFPB contém 119 medicamentos, dos quais 19,3\% e 47,1\% não foram selecionados nas listas de referência nacional e internacional, respectivamente; 16,8\% não são utilizados na atenção primária e 40,3\% não são produzidos por LFO. A revisão do elenco do PFPB com base na concepção de medicamentos essenciais da Organização Mundial da Saúde, o alinhamento às políticas de assistência farmacêutica e à produção pelos LFO são fundamentais para melhorar a qualidade da atenção à saúde, a ges tão, a capacitação dos prescritores e a informação aos cidadãos.

Medicamentos Essenciais; Assistência Farmacêutica; Programas Nacionais de Saúde; Atenção Primária à Saúde 


\section{Introdução}

A Declaração Universal dos Direitos Humanos das Nações Unidas 1 destaca que toda pessoa tem direito a um nível de vida adequado que lhe assegure saúde e bem-estar. Os medicamentos essenciais são bens necessários para a saúde, consequentemente, a Organização Mundial da Saúde (OMS) reconhece seu acesso como um direito humano.

O primeiro posicionamento formal e abrangente do governo brasileiro sobre garantia de acesso a medicamentos essenciais, segundo os critérios da OMS, foi a Política Nacional de Medicamentos 2, cujas diretrizes estão fundamentadas no estabelecimento da Relação Nacional de Medicamentos Essenciais (RENAME). A RENAME deve orientar a produção nacional de medicamentos, a prescrição e o abastecimento, constituindo-se em um mecanismo para a redução dos custos dos medicamentos 2. Alinhandose com a RENAME, a produção dos Laboratórios Farmacêuticos Oficiais do Brasil (LFOB) está direcionada a priorizar os medicamentos essenciais selecionados na RENAME, particularmente os da atenção básica, financiados pelo Sistema Único de Saúde (SUS), para aumentar a concorrência no setor farmacêutico, facilitar o acesso e atuar como referência de preços, custos e qualidade 3 .

Ainda visando à garantia de acesso a medicamentos, a Política Nacional de Assistência Farmacêutica prevê o financiamento daqueles constantes em listas oficiais de medicamentos organizadas em três blocos 4: (i) medicamentos e insumos destinados à atenção básica e aqueles relacionados aos agravos e programas de saúde específicos; (ii) elenco de medicamentos pertencentes aos programas de saúde estratégicos; e (iii) componente especializado, que compreende medicamentos de alto custo cujas linhas de cuidado estão definidas em Protocolos Clínicos e Diretrizes Terapêuticas publicadas pelo Ministério da Saúde 5,6.

Além do financiamento dos três blocos da assistência farmacêutica, existe o Programa Farmácia Popular do Brasil (PFPB) 7, criado em 2004. Esse programa é destinado a ampliar o acesso aos medicamentos considerados básicos e essenciais, de forma igualitária, a qualquer cidadão brasileiro que utilize ou não o sistema público de saúde para o tratamento das doenças e agravos prevalentes. Os medicamentos desse programa podem ser inteiramente financiados pelo governo (elenco subvencionado) ou parcialmente (copagamento).

O PFPB tem seu elenco de medicamentos disponibilizado para a população em um sis- tema de "Rede Própria", constituído por Farmácias Populares, em parceria com os estados, municípios e instituições filantrópicas; ou por meio de convênios com redes privadas de farmácias e drogarias denominadas "Aqui Tem Farmácia Popular" 8.

O abastecimento de medicamentos para Rede Própria é feito prioritariamente a partir dos LFOB 9,10 em compras centralizadas, gerando redução de custos e proporcionando a padronização de produtos e preços em todo o país ${ }^{11}$.

Os medicamentos do elenco da rede Aqui Tem Farmácia Popular são adquiridos diretamente de distribuidoras ou laboratórios farmacêuticos e o valor de venda de cada produto pode variar entre os estabelecimentos conveniados, embora o valor do reembolso pelo governo seja predefinido.

Ponderando os recursos financeiros destinados a esse programa 12, a relevância do mesmo dentro dos três últimos governos brasileiros 13, a priorização de sua produção pelos LFOB 14, as diretrizes norteadoras do programa e a política de medicamentos 2 vigente no país, pretendese analisar o elenco de medicamentos do PFPB quanto à política de assistência farmacêutica 15 vigente, considerando-se três principais aspectos: (i) sua concordância com as listas de medicamentos essenciais da OMS e brasileira, (ii) sua concordância com o componente básico da assistência farmacêutica e a (iii) produção dos LFOB.

\section{Método}

Estudo transversal, descritivo e qualitativo, sobre o elenco de medicamentos do PFPB.

Foram incluídos nesta análise todos os medicamentos constantes nos quatro anexos da Portaria GM/MS no $971^{8}$, de 15 de maio de 2012, que dispõe sobre o PFPB.

Fármacos e medicamentos contidos em mais de uma lista do PFPB foram considerados apenas uma vez. Diferenças nas formas farmacêuticas, bem como as variações nas concentrações foram contabilizadas. Desconsideraram-se apenas as variações de volume nas apresentações comerciais (frasco de $60 \mathrm{~mL}$ ou de $150 \mathrm{~mL}$ da mesma preparação).

A verificação da adequação da nomenclatura dos medicamentos foi feita com base na Denominação Comum Brasileira (DCB) 16. Os fármacos incluídos foram codificados de acordo com a classificação do sistema Anatomical Therapeutic Chemical (ATC), proposto pela OMS (WHO Collaborating Centre for Drug Statistics Methodology. ATC/DDD index 2013. http://www.whocc.no/ atc_ddd_index/, acessado em 30/Abr/2013), em 
13 grupos anatômicos principais 17 e analisados juntamente com suas formas farmacêuticas e concentrações, aqui denominado medicamento.

Na análise, os medicamentos do PFPB contidos nos dois meios de fornecimento (Rede Própria e Aqui Tem Farmácia Popular) foram divididos em dois grupos: (i) aqueles parcialmente financiados pelo governo (copagamento) e; (ii) os subvencionados, ou seja, totalmente subsidiados pelo governo.

Segundo os conceitos da OMS, "medicamentos essenciais são aqueles que servem para satisfazer às necessidades de atenção à saúde da maioria da população. São selecionados de acordo com a sua relevância na saúde pública, evidência sobre a eficácia e a segurança e os estudos comparativos de custo efetividade" 18 (p. 54). Em acordo com esse conceito, a essencialidade foi analisada utilizando-se as listas nacional (RENAME 2010) 19 e internacional (Model List of Essential Medicines - EML 2013) 20. A RENAME 2012 não foi elaborada com base nos critérios de essencialidade da OMS, por isto utilizou-se como referencial, nesta pesquisa, a RENAME 2010.

Além disso, os medicamentos do PFPB que não pertencem à RENAME 2010 e nem à EML 2013 foram comparados com revisões anteriores da RENAME (a partir de 2002) e da EML (a partir de 1995), para analisar se, neste período, houve alguma atualização que o programa desconsiderou.

A verificação da concordância do elenco da PFPB com os componentes da assistência farmacêutica foi feita por meio da comparação com a lista única e geral de medicamentos financiados pelo SUS (RENAME 2012) 21. Em 2012, a RENAME teve suas diretrizes redefinidas pela Resolução no 1/CIT, de 17 de janeiro de 2012 22, que estabelece que "medicamentos essenciais são aqueles definidos pelo SUS para garantir o acesso do usuário ao tratamento medicamentoso" e pela Portaria GM/MS no 533, de 28 de março de 2012 21, que lista o elenco de medicamentos e insumos contidos na RENAME organizados em: (i) Relação Nacional de Medicamentos do Componente Básico da Assistência Farmacêutica; (ii) Relação Nacional de Medicamentos do Componente Estratégico da Assistência Farmacêutica; (iii) Relação Nacional de Medicamentos do Componente Especializado da Assistência Farmacêutica; (iv) Relação Nacional de Medicamentos de Uso Hospitalar; e (v) Relação Nacional de Insumos Farmacêuticos.

A análise do alinhamento do PFPB com a produção de medicamentos nos LFOB foi feita baseando-se em pesquisas de dados sobre o registro dos medicamentos, obtidas entre janeiro de 2012 e abril de 2013, e que estavam dispo- níveis na página da Internet: (i) da Agência Nacional de Vigilância Sanitária (ANVISA; http:// www.anvisa.gov.br/datavisa/consulta_produto/ Medicamentos/frmConsultaMedicamentos.asp, acessado em 30/Abr/2013); (ii) de cada LFOB; e, na falta destas informações, (iii) pelo estudo de Rodrigues 23 que atualizou a lista mediante consulta direta aos LFOB.

\section{Resultados}

Considerando-se a classificação do sistema ATC e excluindo-se as duplicidades de produtos, o elenco do PFPB é composto por 119 medicamentos em 87 códigos ATC diferentes, sendo que 92 (77,3\%) produtos farmacêuticos são financiados por copagamento (Figura 1). A Rede Própria disponibiliza $84,9 \%$ do elenco de medicamentos do PFPB.

Identificou-se que $72,4 \%$ dos medicamentos do PFPB pertencem a cinco grupos anatômicos principais (primeiro nível) da classificação ATC: (i) trato alimentar e metabolismo, (ii) sistema cardiovascular, (iii) anti-infectantes de uso sistêmico, (iv) sistema nervoso e (v) sistema respiratório. Aqueles que pertencem aos grupos: (i) sangue e órgãos hematopoiéticos e (ii) antineoplásicos e agentes imunomoduladores estão presentes em todas as listas, simultaneamente. No grupo $\mathrm{P}$ - antiparasitários, inseticidas e repelentes, encontrou-se maior concordância do PFPB com a EML 2013 do que com a RENAME 2010 (Tabela 1).

A Tabela 2 mostra os medicamentos $(n=19)$ que fazem parte do PFPB e foram excluídos em alguma das revisões da RENAME 2002-2010 (n = 15) e/ou das listas da EML 1995-2013 ( $n=10)$

A Figura 2 indica que $80,7 \%$ do elenco de medicamentos do PFPB estavam também presentes na RENAME 2010, equivalendo a 22 itens subvencionados e 74 financiados por copagamento. Considerando-se essa mesma análise com a EML 2013, foram encontrados 52,9\% de concordância entre as listas, em que 12 dos medicamentos são subvencionados e 51 fornecidos por copagamento.

Comparando-se o PFPB com o componente básico da assistência farmacêutica foram identificados 22 medicamentos subvencionados, 80 financiados por copagamento e 20 do PFPB que não pertencem somente ao componente básico da assistência farmacêutica, dos quais: 1,7\% faz parte somente do elenco de medicamentos do componente especializado (sulfassalazina $500 \mathrm{mg}$ e azatioprina $50 \mathrm{mg}$ ) e $0,8 \%$ pertence ao componente estratégico (doxiciclina 100mg) (Figura 2). 
Diagrama de composição do elenco de medicamentos do PFPB, com base na Portaria GM/MS no 9718.

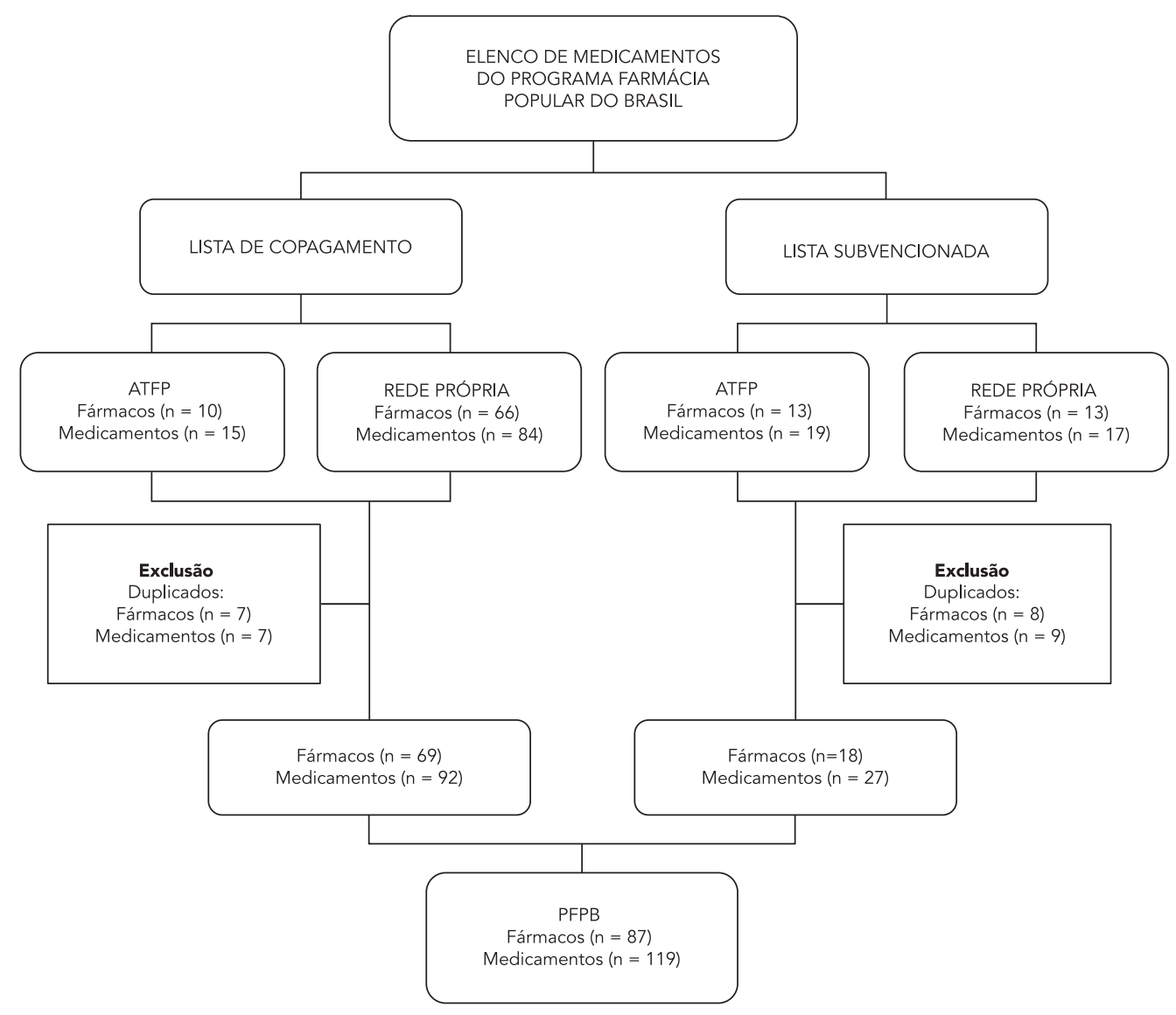

PFPB: Programa Farmácia Popular do Brasil.

A Tabela 3 contém informações detalhadas do elenco de medicamentos do PFPB comparando-os à RENAME 2010, à EML 2013, à produção dos LFOB e à RENAME 2012. Quanto à capacidade dos LFOB para suprir o PFPB, foi observado que eles produzem 71 (59,7\%) dos 119 medicamentos existentes em todo o programa, mas, 33 $(27,7 \%)$ e 15 (12,6\%) medicamentos produzidos não constam na EML 2013 e na RENAME 2010, respectivamente. Análise mais detalhada mostra que os LFOB produzem 69 (68,3 \%) medicamentos da Rede Própria, sendo $12(70,6 \%)$ dos itens subvencionados e $57(67,9 \%)$ financiados por copagamento. Os LFOB não produzem os hormônios contidos no PFPB ( $n=5)$, nem as formas farmacêuticas que requeiram dispositivo para inalar ou aspirar $(\mathrm{n}=11)$, como as utilizadas no tratamento das doenças respiratórias.

\section{Discussão}

Esta pesquisa avalia a seleção de medicamentos do PFPB comparando-a aos preceitos da política de assistência farmacêutica no Brasil 15 . O elenco do programa foi avaliado segundo critérios de essencialidade, alinhamento aos componentes da assistência farmacêutica e concordância com a produção de medicamentos pelos LFOB.

O PFPB foi proposto em resposta à baixa disponibilidade demedicamentos na rede pública 10 , e para garantir o acesso da população àqueles considerados essenciais e pertencentes à atenção primária ${ }^{8}$.

O elenco de medicamentos do PFPB apresenta medicamentos não essenciais quando comparado com a RENAME 2010 (19,3\%) e com a EML 
Tabela 1

Distribuição ( $\mathrm{n}$ \% \%) de fármacos e medicamentos concordantes com o PFPB, segundo grupo anatômico principal (ATC), nas listas de medicamentos essenciais da Organização Mundial da Saúde (EML 2013) e do Brasil (RENAME 2010) e contemplados na produção pelos LFOB. Brasil, 2014.

\begin{tabular}{|c|c|c|c|c|c|c|c|c|}
\hline \multirow[t]{2}{*}{ ATC * } & \multicolumn{2}{|c|}{ PFPB } & \multicolumn{2}{|c|}{ RENAME 2010} & \multicolumn{2}{|c|}{ EML 2013} & \multicolumn{2}{|c|}{ LFOB } \\
\hline & $\begin{array}{c}\text { Fármaco } \\
\mathbf{n}\end{array}$ & $\begin{array}{c}\text { Medicamento } \\
\mathbf{n}\end{array}$ & $\begin{array}{c}\text { Fármaco } \\
\text { n (\%) }\end{array}$ & $\begin{array}{c}\text { Medicamento } \\
\text { n (\%) }\end{array}$ & $\begin{array}{c}\text { Fármaco } \\
\text { n (\%) }\end{array}$ & $\begin{array}{c}\text { Medicamento } \\
\text { n (\%) }\end{array}$ & $\begin{array}{c}\text { Fármaco } \\
\text { n (\%) }\end{array}$ & $\begin{array}{c}\text { Medicamento } \\
n(\%)\end{array}$ \\
\hline$A$ & 12 & 14 & $10(83,3)$ & $12(85,7)$ & $9(75,0)$ & $9(64,3)$ & $8(66,7)$ & $9(64,3)$ \\
\hline$B$ & 3 & 4 & $3(100,0)$ & $4(100,0)$ & $3(100,0)$ & $3(75,0)$ & $3(100,0)$ & $4(100,0)$ \\
\hline C & 14 & 18 & $13(92,9)$ & $14(77,8)$ & $8(57,1)$ & $10(55,6)$ & $8(57,1)$ & $10(55,6)$ \\
\hline D & 4 & 5 & $2(50,0)$ & $3(60,0)$ & $1(25,0)$ & $1(20,0)$ & $2(50,0)$ & $2(40,0)$ \\
\hline G & 7 & 7 & $6(85,7)$ & $4(57,1)$ & $3(42,9)$ & $3(42,9)$ & $1(14,3)$ & $1(14,3)$ \\
\hline $\mathrm{H}$ & 1 & 2 & $1(100,0)$ & $2(100,0)$ & $0(0,0)$ & $0(0,0)$ & $1(100,0)$ & $2(100,0)$ \\
\hline$J$ & 12 & 17 & $11(91,7)$ & $16(94,1)$ & $8(66,7)$ & $10(58,8)$ & $11(91,7)$ & $14(82,3)$ \\
\hline L & 1 & 1 & $1(100,0)$ & $1(100,0)$ & $1(100,0)$ & $1(100,0)$ & $1(100,0)$ & $1(100,0)$ \\
\hline M & 3 & 3 & $3(100,0)$ & $3(100,0)$ & $1(66,7)$ & $1(33,3)$ & $2(66,7)$ & $2(66,7)$ \\
\hline $\mathrm{N}$ & 15 & 22 & $15(100,0)$ & $18(81,8)$ & $12(80,0)$ & $15(68,2)$ & $11(73,3)$ & $16(72,7)$ \\
\hline$P$ & 4 & 6 & $2(50,0)$ & $3(50,0)$ & $3(75,0)$ & $4(66,7)$ & $2(50,0)$ & $4(66,7)$ \\
\hline $\mathrm{R}$ & 10 & 18 & $10(100,0)$ & $14(77,8)$ & $3(30,0)$ & $4(22,2)$ & $5(50,0)$ & $6(33,3)$ \\
\hline S & 1 & 2 & $1(100,0)$ & $2(100,0)$ & $1(100,0)$ & $2(100,0)$ & $0(0,0)$ & $0(0,0)$ \\
\hline Total & 87 & 119 & $78(89,7)$ & $96(80,7)$ & $53(60,9)$ & $63(52,9)$ & $55(63,2)$ & $71(59,7)$ \\
\hline
\end{tabular}

ATC: Anatomical Therapeutic Chemical; EML: Model List of Essential Medicines; LFOB: Laboratórios Farmacêuticos Oficiais do Brasil;

PFPB: Programa Farmácia Popular do Brasil; RENAME: Relação Nacional de Medicamentos Essenciais.

* A: trato alimentar e metabolismo; B: sangue e órgãos hematopoiéticos; C: sistema cardiovascular; D: dermatológicos; G: sistema geniturinário e hormônios sexuais; H: compostos hormonais sistêmicos, excluindo hormônios sexuais e insulina; J: anti-infecciosos de uso sistêmico; L: antineoplásicos e agentes imunomoduladores; M: sistema muscular esquelético; N: sistema nervoso; P: antiparasitários, inseticidas e repelentes; R: sistema respiratório; S: sistema ocular.

2013 (47,1\%) (Tabela 1). Dos 23 medicamentos do Programa não pertencentes à RENAME 2010, cinco deles também não constam na EML 2013. Nesta mesma análise, verificou-se que as listas de copagamento e daqueles subvencionados do PFPB (Figura 2) possuem, em média, 16\% do seu elenco classificados como não essenciais (Tabela 3 ).

A análise deste elenco revela a presença de medicamentos que já foram excluídos em edições anteriores das listas de referência (nacional e internacional), ou que nunca foram incluídos nas mesmas por não atenderem aos critérios de essencialidade. Este programa existe há dez anos, concomitantemente a sucessivas revisões das listas de medicamentos essenciais do Brasil e da OMS, o que não justifica a presença de itens não essenciais em seu elenco. Ainda demonstra a inexistência de revisão permanente ou periódica de seu elenco como está descrito na Portaria GM/ MS no 971 8, problema este detectado por Pinto 24, mas não solucionado.

Apesar da alteração do conceito de medicamento essencial na RENAME em 2012 (Resolução no 1/CIT 22 e Portaria GM/MS no 533 21), os preceitos do PFPB se basearam no conceito da essencialidade, definido pela OMS (uma vez que sua criação antecede 2012), mas suas atualizações não seguiram tais critérios. Não está claro o critério que define a inclusão ou exclusão de um medicamento neste Programa. Além disso, alterações no elenco de medicamentos do PFPB deveriam ser balizadas em justificativas técnicopolíticas e publicadas na forma de pareceres ou de notas técnicas à comunidade, o que não vem acontecendo.

Os LFOB produzem 59,7\% dos medicamentos incluídos no PFPB. Como somente a Rede Própria pode comprar desses laboratórios, isso significa que $70,6 \%$ do elenco subvencionado e $67,9 \%$ do copagamento da Rede Própria estão sendo produzidos ou podem ser supridos pelos mesmos. Esses valores, embora significativos, não são ideais, pois, trata-se de uma lista de medicamentos para a atenção básica e, portanto, deveriam ser prioridades em políticas públicas de saúde. Isso mostra que os LFOB ainda estão aquém de conseguir alinhar sua produção com as necessidades básicas da população em termos de medicamentos.

A presença de um medicamento no elenco do PFPB pode determinar a sua prioridade de produção pelos LFOB, e a existência de problemas 
Tabela 2

Relação de medicamentos excluídos em alguma das revisões da RENAME e/ou da EML e que constam no elenco do PFPB.

\begin{tabular}{|c|c|c|c|c|c|}
\hline ATC & Fármaco & Dose/Concentração & Unidade de produto & RENAME & EML \\
\hline А03BB01 & Butilbrometo de escopolamina * & $10 \mathrm{mg} / \mathrm{mL}$ & Solução oral (gotas) & Excluído em 2006 & Inexistente \\
\hline А10BB01 & Glibenclamida & $5 \mathrm{mg}$ & Comprimido & Consta & Excluído em 2013 \\
\hline A12BA01 & Cloreto de potássio & $60 \mathrm{mg} / \mathrm{mL}$ & Xarope & Excluído em 2006 & Inexistente \\
\hline С07АB03 & Atenolol & $25 \mathrm{mg}$ & Comprimido & Consta & Excluído em 2011 \\
\hline C08CA06 & Nifedipino ** & $20 \mathrm{mg}$ & $\begin{array}{l}\text { Comprimido de liberação } \\
\text { contínua }\end{array}$ & Excluído em 2006 & Inexistente \\
\hline C09AA01 & Captopril & $25 \mathrm{mg}$ & Comprimido & Consta & Excluído em 2003 \\
\hline D01AC06 & Tiabendazol & $5 \%$ & Pomada & Excluído em 2006 & Excluído em 1995 \\
\hline D06AX & $\begin{array}{c}\text { Sulfato de neomicina + } \\
\text { bacitracina zíncica }\end{array}$ & $(5 \mathrm{mg}+250 \mathrm{UI}) / \mathrm{g}$ & Pomada & Excluído em 2006 & Excluído em 2011 \\
\hline G01AA01 & Nistatina & $25.000 U 1$ & Creme vaginal & Excluído em 2006 & Inexistente \\
\hline G01AF01 & 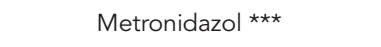 & $5 \%$ & Creme vaginal & Excluído em 2008 & Excluído em 2009 \\
\hline J02AB02 & Cetoconazol & $200 \mathrm{mg}$ & Comprimido & Excluído em 2006 & Excluído em 2000 \\
\hline N02BB02 & Dipirona & $500 \mathrm{mg} / \mathrm{mL}$ & Solução oral (gotas) & Excluído em 2010 & Inexistente \\
\hline N03AE01 & Clonazepam & $2 \mathrm{mg}$ & Comprimido & Consta & Excluído em 2005 \\
\hline P02CA01 & Mebendazol & $100 \mathrm{mg}$ & Comprimido & Excluído em 2010 & Consta \\
\hline P02CA01 & Mebendazol & $20 \mathrm{mg} / \mathrm{mL}$ & Suspensão oral & Excluído em 2010 & Inexistente \\
\hline P03AX01 & Benzoato de benzila\# & $200 \mathrm{mg} / \mathrm{mL}$ & Emulsão & Excluído em 2006 & Inexistente \\
\hline $\mathrm{RO} 3 \mathrm{CCO} 2$ & Sulfato de salbutamol & $2 \mathrm{mg}$ & Comprimido sulcado & Excluído em 2006 & Excluído em 2011 \\
\hline $\mathrm{R03CCO2}$ & Sulfato de salbutamol & $0,4 \mathrm{mg} / \mathrm{mL}$ & Xarope & Excluído em 2006 & Inexistente \\
\hline R06AD02 & Cloridrato de prometazina & $25 \mathrm{mg}$ & Comprimido & Excluído em 2006 & Excluído em 2011 \\
\hline
\end{tabular}

EML: Model List of Essential Medicines; PFPB: Programa Farmácia Popular do Brasil; RENAME: Relação Nacional de Medicamentos Essenciais.

* Butilbrometo de escopolamina: fármaco excluído na RENAME 2006, cuja forma farmacêutica era distinta (injetável e comprimido);

** Nifedipino: fármaco excluído da RENAME 2006 como antianginoso e incluído como tocolítico;

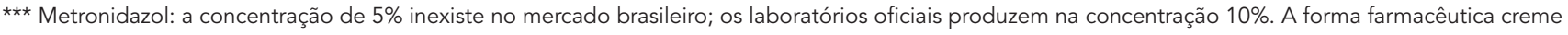
vaginal é comercializada por dois laboratórios, e são comercializados mais de 20 medicamentos na forma de gel vaginal;

\# Benzoato de benzila: a concentração $200 \mathrm{mg} / \mathrm{mL}$ não é comercializada no Brasil ou no exterior; os laboratórios farmacêuticos oficiais produzem na concentração $250 \mathrm{mg} / \mathrm{mL}$.

relacionados à sua seleção leva a uma sucessão de erros no ciclo da AF, inclusive fomentando a produção de medicamentos não essenciais. Vale citar o caso daqueles que não atendem aos critérios de essencialidade da OMS e que foram excluídos em edições anteriores da RENAME ou da EML: (i) sulfato de neomicina + bacitracina zíncica $(5 \mathrm{mg}+250 \mathrm{UI}) / \mathrm{g}$ pomada, (ii) cetoconazol 200mg comprimido, (iii) salbutamol 0,4mg/ $\mathrm{mL}$ xarope, (iv) salbutamol $2 \mathrm{mg}$ comprimido, $\mathrm{v}$. nistatina creme vaginal $25.000 \mathrm{UI} / \mathrm{g}$ e (vi) mebendazol suspensão oral 20mg/mL.

Apesar desses equívocos, os LFOB atuam como reguladores de preços no mercado nacional e são utilizados como estratégia na indução de políticas de assistência farmacêutica 25 , a fim de facilitar o acesso a medicamentos.

A presença, no elenco do PFPB, de formas farmacêuticas não registradas na ANVISA e que, portanto, não deveriam ser produzidas ou co- mercializadas no país, causa estranheza, uma vez que este é o critério utilizado nas licitações de aquisição de medicamentos no SUS. Essa última análise pode estar limitada pela falta de atualização de dados nos sítios eletrônicos da ANVISA e dos próprios laboratórios quanto ao que está registrado e sendo produzido. Para superar essas limitações foram utilizados dados de três fontes, conforme descrito no método.

Segundo informações disponíveis para consulta na base de dados da ANVISA, o metronidazol na forma de creme vaginal $5 \%$, presente no elenco do PFPB, não possui registro para comercialização, a concentração do fármaco que possui registro e comercialização no mercado brasileiro é a de 10\%; ou mesmo benzoato de benzila na forma de suspensão de $200 \mathrm{mg} / \mathrm{mL}$, que apesar de não ser considerado essencial desde a revisão da RENAME 2006, tem registro e comercialização apenas na concentração de 
Comparação da porcentagem de medicamentos do PFPB que estão presentes nas listas de referência nacional (RENAME 2010) e internacional (EML 2013), nos registros de medicamentos produzidos pelos LFOB e na lista dos componentes da assistência farmacêutica (RENAME 2012).

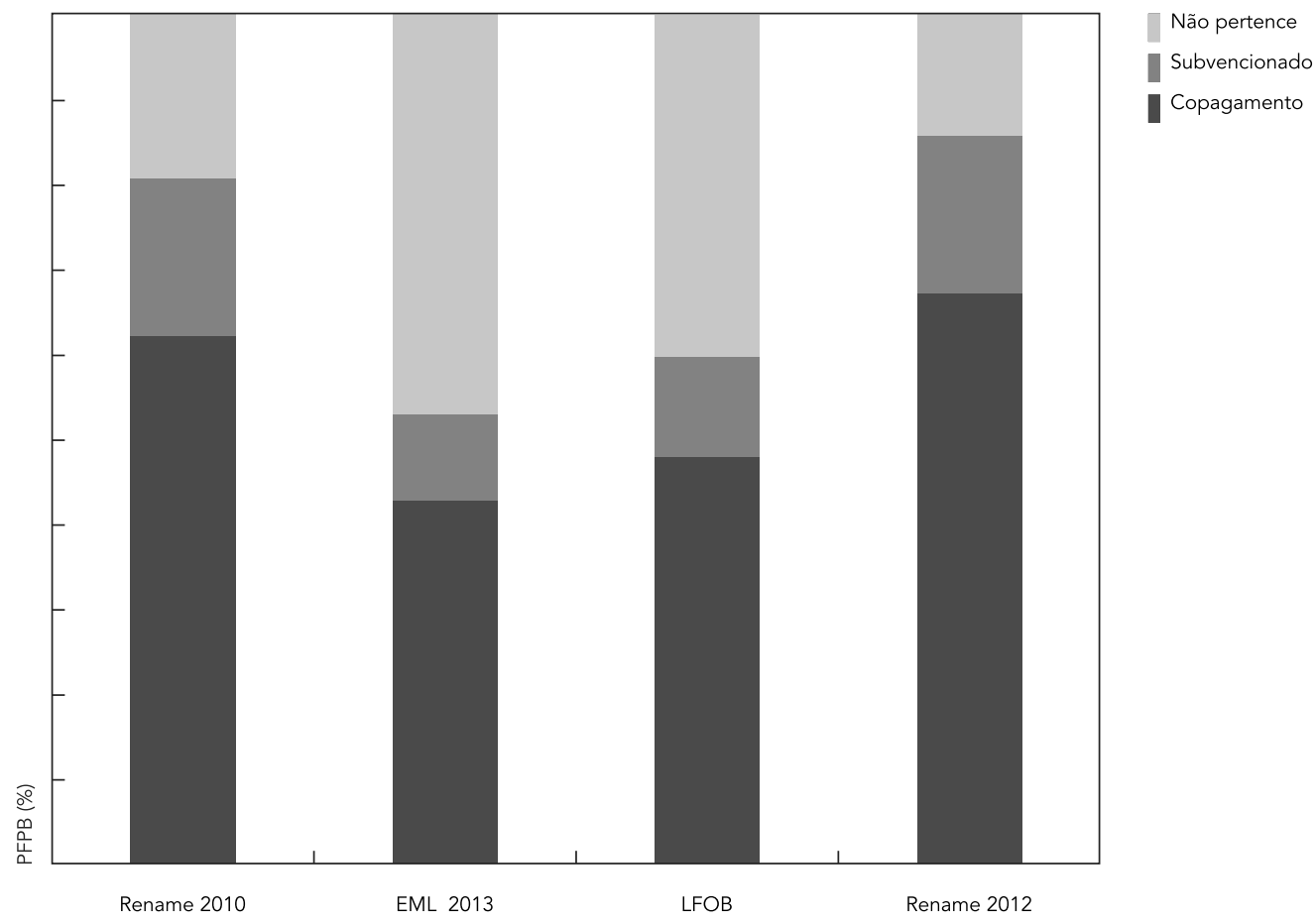

EML: Model List of Essential Medicines; LFOB: Laboratórios Farmacêuticos Oficiais do Brasil; PFPB: Programa Farmácia Popular do Brasil; RENAME: Relação Nacional de Medicamentos Essenciais.

250mg/mL. Fato interessante é o que ocorre com o sal de eritromicina, pois desde 2011 todos os registros de estearato de eritromicina (sal incluído no PFPB) na ANVISA estão vencidos e há registro apenas de estolato de eritromicina. Etilsuccinato de eritromicina, também incluído na lista do $\mathrm{PFPB}$, nunca foi registrado no Brasil. Como esses produtos podem constar no PFPB se realmente não estiverem autorizados para distribuição ou comercialização no país?

Verificou-se ainda que a lista de medicamentos do PFPB não se destina somente à atenção primária, conforme definido na Portaria GM no 971 , de 15 de maio de $2012{ }^{8}$, pois $16,8 \%$ dos medicamentos pertencem a outros componentes da AF: sulfassalazina e a azatioprina (componente especializado) e doxiciclina (componente estratégico), ademais, nenhum destes medicamentos é produzido por LFOB.
Medicamentos do componente especializado possuem protocolos clínicos para acompanhamento dos pacientes, além de critérios e locais específicos de dispensação (farmácias mantidas pela Secretaria de Estado da Saúde); e medicamentos do componente estratégico são utilizados para o controle de endemias, além de serem adquiridos pela União. Incluí-los numa lista de medicamentos básicos com fornecimento vinculado somente à prescrição reflete o contraditório na política de medicamentos no Brasil.

Medicamentos indicados para situações clínicas complexas, que apresentam custo elevado, devem ser prescritos segundo condições específicas do paciente e seguir fluxograma de tratamento que considere resultados de exames e evolução da doença, com seguimento farmacoterapêutico adequado, em ambulatórios especializados, a fim de evitar o aparecimento de eventos adversos graves e uso inadequado do medicamento 6 . 
Tabela 3

Presença de medicamentos constantes no PFPB, em listas de referência nacional (RENAME 2010) e internacional (EML 2013), na lista de produção de LFOB e na lista dos componentes de financiamento da assistência farmacêutica.

\begin{tabular}{|c|c|c|c|c|c|c|c|}
\hline Medicamento & ATC & Dose/Concentração & $\begin{array}{c}\text { Forma } \\
\text { farmacêutica }\end{array}$ & $\begin{array}{l}\text { RENAME } \\
2010\end{array}$ & $\begin{array}{l}\text { EML } \\
2013\end{array}$ & LFOB & CAF \\
\hline \multicolumn{8}{|c|}{ Trato alimentar e metabolismo (ATC - A) } \\
\hline Ranitidina & A02BA02 & $150 \mathrm{mg}$ & Comprimido & $\mathrm{S}$ & $\mathrm{S}$ & $\mathrm{S}$ & CBAF \\
\hline Omeprazol & A02BC01 & $20 \mathrm{mg}$ & Cápsula & $\mathrm{S}$ & $\mathrm{S}$ & $\mathrm{N}$ & CBAF \\
\hline Butilbrometo de escopolamina & А03BВ01 & $10 \mathrm{mg} / \mathrm{mL}$ & Solução oral (gotas) & $\mathrm{N}$ & $\mathrm{N}$ & $\mathrm{N}$ & NA \\
\hline \multirow[t]{2}{*}{ Cloridrato de metoclopramida } & A03FA01 & $10 \mathrm{mg}$ & Comprimido & $\mathrm{S}$ & $\mathrm{S}$ & $\mathrm{S}$ & CBAF \\
\hline & & $4 \mathrm{mg} / \mathrm{mL}$ & Solução oral (gotas) & $\mathrm{S}$ & $\mathrm{N}$ & $\mathrm{S}$ & CBAF \\
\hline Nistatina & A07AA02 & $100.000 U \mathrm{I} / \mathrm{mL}$ & Suspensão oral & $\mathrm{S}$ & $\mathrm{S}$ & $\mathrm{N}$ & CBAF \\
\hline Sais para reidratação oral & A07CA & $27,9 \mathrm{~g}$ & Pó para suspensão oral & $\mathrm{S}$ & $\mathrm{S}$ & $\mathrm{S}$ & CBAF \\
\hline Sulfassalazina & A07EC01 & $500 \mathrm{mg}$ & Comprimido & $\mathrm{S}$ & $\mathrm{S}$ & $N$ & CEAF \\
\hline Insulina humana regular * & A10AB01 & $100 \mathrm{UI} / \mathrm{mL}$ & Solução injetável & $\mathrm{S}$ & $\mathrm{S}$ & $\mathrm{S}$ & CBAF \\
\hline Insulina humana NPH * & A10AC01 & $100 \mathrm{UI} / \mathrm{mL}$ & Suspensão injetável & $\mathrm{S}$ & $\mathrm{S}$ & $\mathrm{S}$ & CBAF \\
\hline \multirow[t]{2}{*}{ Cloridrato de metformina * } & A10BA02 & $500 \mathrm{mg}$ & Comprimido & $\mathrm{S}$ & $\mathrm{S}$ & $\mathrm{N}$ & CBAF \\
\hline & & $850 \mathrm{mg}$ & & $\mathrm{S}$ & $\mathrm{N}$ & $\mathrm{S}$ & CBAF \\
\hline Glibenclamida * & A10BB01 & $5 \mathrm{mg}$ & Comprimido & $\mathrm{S}$ & $\mathrm{N}$ & $\mathrm{S}$ & CBAF \\
\hline Cloreto de potássio & A12BA01 & $60 \mathrm{mg} / \mathrm{mL}$ & Xarope & $\mathrm{N}$ & $\mathrm{N}$ & $\mathrm{S}$ & NA \\
\hline Subtotal & 12 & 14 & & 12 & 9 & 9 & 12 \\
\hline \multicolumn{8}{|c|}{ Sangue e órgãos hematopoiéticos (ATC - B) } \\
\hline Ácido acetilsalicílico & $\mathrm{B} 01 \mathrm{AC} 06$ & $100 \mathrm{mg}$ & Comprimido & $\mathrm{S}$ & $\mathrm{S}$ & $\mathrm{S}$ & CBAF \\
\hline \multirow[t]{2}{*}{ Sulfato ferroso } & B03AA07 & 40mg Fe(II) & Comprimido revestido & $\mathrm{S}$ & $\mathrm{N}$ & $\mathrm{S}$ & CBAF \\
\hline & & $25 \mathrm{mg} / \mathrm{mL} \mathrm{Fe}(\mathrm{II})$ & Solução oral (gotas) & $\mathrm{S}$ & $\mathrm{S}$ & $\mathrm{S}$ & CBAF \\
\hline Ácido fólico & В03ВВ01 & $5 \mathrm{mg}$ & Comprimido & $\mathrm{S}$ & $\mathrm{S}$ & $\mathrm{S}$ & CBAF \\
\hline Subtotal & 3 & 4 & & 4 & 3 & 4 & 4 \\
\hline \multicolumn{8}{|l|}{ Sistema cardiovascular (ATC - C) } \\
\hline Digoxina & C01AA05 & $0,25 \mathrm{mg}$ & Comprimido & $\mathrm{S}$ & $\mathrm{S}$ & $\mathrm{S}$ & CBAF \\
\hline Amiodarona & C01BD01 & $200 \mathrm{mg}$ & Comprimido & $\mathrm{S}$ & $\mathrm{S}$ & $\mathrm{N}$ & CBAF \\
\hline Mononitrato de isossorbida & C01DA14 & $20 \mathrm{mg}$ & Comprimido & $\mathrm{N}$ & $\mathrm{N}$ & $\mathrm{S}$ & CBAF \\
\hline \multirow[t]{2}{*}{ Metildopa * } & С02АB01 & $250 \mathrm{mg}$ & Comprimido revestido & $\mathrm{S}$ & S & $\mathrm{S}$ & CBAF \\
\hline & & $500 \mathrm{mg}$ & & $N$ & $\mathrm{~N}$ & $\mathrm{~S}$ & NA \\
\hline Hidroclorotiazida * & C03AA03 & $25 \mathrm{mg}$ & Comprimido & $\mathrm{S}$ & $\mathrm{S}$ & $\mathrm{S}$ & CBAF \\
\hline Furosemida * & C03CA01 & $40 \mathrm{mg}$ & Comprimido & $\mathrm{S}$ & S & $\mathrm{S}$ & CBAF \\
\hline Cloridrato de propranolol * & C07AA05 & $40 \mathrm{mg}$ & Comprimido & $\mathrm{S}$ & $\mathrm{S}$ & $\mathrm{S}$ & CBAF \\
\hline Atenolol * & С07АBO3 & $25 \mathrm{mg}$ & Comprimido & $\mathrm{N}$ & $N$ & $\mathrm{~N}$ & NA \\
\hline Nifedipino * & C08CA06 & $20 \mathrm{mg}$ & $\begin{array}{l}\text { Comprimido de } \\
\text { liberação contínua }\end{array}$ & $\mathrm{N}$ & $\mathrm{N}$ & $\mathrm{S}$ & NA \\
\hline Cloridrato de verapamil * & C08DA01 & $80 \mathrm{mg}$ & Comprimido & $\mathrm{S}$ & $\mathrm{S}$ & $N$ & CBAF \\
\hline Captopril * & C09AA01 & $25 \mathrm{mg}$ & Comprimido & $\mathrm{S}$ & $\mathrm{N}$ & $\mathrm{S}$ & CBAF \\
\hline Enalapril * & C09AA02 & $20 \mathrm{mg}$ & Comprimido & $\mathrm{S}$ & $\mathrm{N}$ & $\mathrm{S}$ & CBAF \\
\hline Maleato de enalapril * & CO9AA02 & $10 \mathrm{mg}$ & Comprimido & $\mathrm{S}$ & $\mathrm{N}$ & $\mathrm{N}$ & CBAF \\
\hline Losartana potássica * & C09CA01 & $50 \mathrm{mg}$ & Comprimido & $\mathrm{S}$ & $\mathrm{N}$ & $\mathrm{N}$ & CBAF \\
\hline \multirow[t]{3}{*}{ Sinvastatina } & C10AA01 & $10 \mathrm{mg}$ & Comprimido & $\mathrm{S}$ & $\mathrm{S}$ & $N$ & CBAF \\
\hline & & $20 \mathrm{mg}$ & & $\mathrm{S}$ & $\mathrm{S}$ & $N$ & CBAF \\
\hline & & $40 \mathrm{mg}$ & & $\mathrm{S}$ & $\mathrm{S}$ & $\mathrm{N}$ & CBAF \\
\hline Subtotal & 14 & 18 & & 14 & 10 & 10 & 15 \\
\hline
\end{tabular}

(continua) 


\begin{tabular}{|c|c|c|c|c|c|c|c|}
\hline Medicamento & ATC & Dose/Concentração & $\begin{array}{c}\text { Forma } \\
\text { farmacêutica }\end{array}$ & $\begin{array}{l}\text { RENAME } \\
2010\end{array}$ & $\begin{array}{l}\text { EML } \\
2013\end{array}$ & LFOB & CAF \\
\hline \multicolumn{8}{|l|}{ Dermatológicos (ATC - D) } \\
\hline \multirow[t]{2}{*}{ Nitrato de miconazol } & D01AC02 & $2 \%$ & Loção & $\mathrm{S}$ & $\mathrm{S}$ & $\mathrm{N}$ & CBAF \\
\hline & & $2 \%$ & Pó & $\mathrm{S}$ & $\mathrm{N}$ & $\mathrm{N}$ & CBAF \\
\hline Tiabendazol & D01AC06 & $5 \%$ & Pomada & $\mathrm{N}$ & $\mathrm{N}$ & $\mathrm{N}$ & NA \\
\hline Sulfato de neomicina + bacitracina zíncica & D06AX & $(5 \mathrm{mg}+250 U \mathrm{I}) / \mathrm{g}$ & Pomada & $\mathrm{N}$ & $\mathrm{N}$ & $\mathrm{S}$ & NA \\
\hline Dexametasona & D07AB19 & $0,10 \%$ & Creme & $\mathrm{S}$ & $\mathrm{N}$ & $\mathrm{S}$ & CBAF \\
\hline Subtotal & 4 & 5 & & 3 & 1 & 2 & 3 \\
\hline \multirow{2}{*}{\multicolumn{8}{|c|}{$\begin{array}{l}\text { Sistema geniturinário e hormônios sexuais } \\
\text { (ATC - G) }\end{array}$}} \\
\hline & & & & & & & \\
\hline Nistatina & G01AA01 & $25.000 U 1$ & Creme vaginal & $\mathrm{N}$ & $\mathrm{N}$ & $\mathrm{S}$ & NA \\
\hline Metronidazol & G01AF01 & $5 \%$ & Creme vaginal & $\mathrm{N}$ & $\mathrm{N}$ & $\mathrm{N}$ & NA \\
\hline $\begin{array}{l}\text { Enantato de noretisterona + valerato de } \\
\text { estradiol }\end{array}$ & G03AA & $50 m g+5 m g$ & Injetável & $\mathrm{S}$ & $\mathrm{N}$ & $\mathrm{N}$ & CBAF \\
\hline Etinilestradiol + levonorgestrel & G03AA07 & $0,03 m g+0,15 m g$ & Comprimido & $\mathrm{S}$ & $\mathrm{S}$ & $\mathrm{N}$ & CBAF \\
\hline Norestisterona & G03AC01 & $0,35 \mathrm{mg}$ & Comprimido & $\mathrm{S}$ & $\mathrm{N}$ & $\mathrm{N}$ & CBAF \\
\hline Levonorgestrel & G03AC03 & $0,75 \mathrm{mg}$ & Comprimido & $\mathrm{N}$ & $\mathrm{S}$ & $\mathrm{N}$ & CBAF \\
\hline Acetato de medroxiprogesterona & G03AC06 & $150 \mathrm{mg} / \mathrm{mL}$ & Injetável & $\mathrm{S}$ & $\mathrm{S}$ & $\mathrm{N}$ & CBAF \\
\hline Subtotal & 7 & 7 & & 4 & 3 & 1 & 5 \\
\hline \multicolumn{8}{|l|}{$\begin{array}{l}\text { Compostos hormonais sistêmicos, excluindo } \\
\text { hormônios sexuais e insulina (ATC - H) }\end{array}$} \\
\hline \multirow[t]{4}{*}{ Prednisona } & $\mathrm{H} 02 \mathrm{AB} 07$ & $20 \mathrm{mg}$ & Comprimido & $\mathrm{S}$ & $\mathrm{N}$ & $\mathrm{S}$ & CBAF/ \\
\hline & & & & & & & CESAF \\
\hline & & $5 \mathrm{mg}$ & & $\mathrm{S}$ & $\mathrm{N}$ & $\mathrm{S}$ & CBAF/ \\
\hline & & & & & & & CESAF \\
\hline Subtotal & 1 & 2 & & 2 & 0 & 2 & 2 \\
\hline \multicolumn{8}{|l|}{ Anti-infecciosos de uso sistêmico (ATC - J) } \\
\hline Doxiciclina & J01AA02 & $100 \mathrm{mg}$ & Comprimido & $\mathrm{S}$ & $\mathrm{S}$ & $\mathrm{S}$ & CESAF \\
\hline \multirow[t]{2}{*}{ Amoxicilina } & J01CA04 & $500 \mathrm{mg}$ & Cápsula & $\mathrm{S}$ & $\mathrm{S}$ & $\mathrm{S}$ & CBAF \\
\hline & & $50 \mathrm{mg} / \mathrm{mL}$ & Pó para suspensão oral & $\mathrm{S}$ & $\mathrm{S}$ & $\mathrm{S}$ & CBAF \\
\hline Benzilpenicilina benzatina & J01CE08 & $1.200 .000 U 1$ & $\begin{array}{l}\text { Pó para suspensão } \\
\text { injetável }\end{array}$ & $\mathrm{S}$ & $\mathrm{S}$ & $\mathrm{S}$ & CBAF \\
\hline Benzilpenicilina procaína + potássica & J01CE30 & $300.000+100.000 U 1$ & $\begin{array}{l}\text { Pó para suspensão } \\
\text { injetável }\end{array}$ & $\mathrm{S}$ & $\mathrm{N}$ & $\mathrm{S}$ & CBAF \\
\hline \multirow[t]{2}{*}{ Cefalexina (cloridrato ou sal sódico) } & J01DB01 & $500 \mathrm{mg}$ & Cápsula & $\mathrm{S}$ & $\mathrm{N}$ & $\mathrm{S}$ & CBAF \\
\hline & & $50 \mathrm{mg} / \mathrm{mL}$ & Pó para suspensão oral & $\mathrm{S}$ & $\mathrm{S}$ & $\mathrm{N}$ & CBAF \\
\hline \multirow[t]{2}{*}{ Sulfametoxazol + trimetoprima } & J01EE01 & $400 m g+80 m g$ & Comprimido & $\mathrm{S}$ & $\mathrm{S}$ & $\mathrm{S}$ & CBAF \\
\hline & & $(40 m g+8 m g) / m L$ & Suspensão oral & $\mathrm{S}$ & $\mathrm{S}$ & $\mathrm{S}$ & CBAF \\
\hline \multirow[t]{4}{*}{ Eritromicina (estearato ou etilsuccinato) } & J01FA01 & $25 \mathrm{mg} / \mathrm{mL}$ & Pó para suspensão oral & $\mathrm{S}$ & $\mathrm{S}$ & $\mathrm{S}$ & CBAF/ \\
\hline & & & & & & & CESAF \\
\hline & & $500 \mathrm{mg}$ & Comprimido ou cápsula & $\mathrm{S}$ & $\mathrm{N}$ & $\mathrm{S}$ & CBAF/ \\
\hline & & & & & & & CESAF \\
\hline \multirow[t]{2}{*}{ Azitromicina } & J01FA10 & $500 \mathrm{mg}$ & Comprimido & $\mathrm{S}$ & $\mathrm{S}$ & $\mathrm{N}$ & CBAF/ \\
\hline & & & & & & & CESAF \\
\hline Ciprofloxacino & J01MA02 & $500 \mathrm{mg}$ & Comprimido & $\mathrm{S}$ & $\mathrm{N}$ & $\mathrm{S}$ & CBAF \\
\hline Cetoconazol & J02AB02 & $200 \mathrm{mg}$ & Comprimido & $\mathrm{N}$ & $\mathrm{N}$ & $\mathrm{S}$ & NA \\
\hline \multirow[t]{3}{*}{ Fluconazol } & J02AC01 & $100 \mathrm{mg}$ & Cápsula revestida & $\mathrm{S}$ & $\mathrm{N}$ & S & CBAF/ \\
\hline & & & & & & & CESAF \\
\hline & & $150 \mathrm{mg}$ & Cápsula revestida & $\mathrm{S}$ & $\mathrm{N}$ & $\mathrm{N}$ & CBAF \\
\hline Aciclovir & J05AB01 & $200 \mathrm{mg}$ & Comprimido & $\mathrm{S}$ & $\mathrm{S}$ & $\mathrm{S}$ & CBAF \\
\hline Subtotal & 12 & 17 & & 16 & 10 & 14 & 16 \\
\hline
\end{tabular}

(continua) 
Tabela 3 (continuação)

\begin{tabular}{|c|c|c|c|c|c|c|c|}
\hline Medicamento & ATC & Dose/Concentração & $\begin{array}{c}\text { Forma } \\
\text { farmacêutica }\end{array}$ & $\begin{array}{l}\text { RENAME } \\
2010\end{array}$ & $\begin{array}{l}\text { EML } \\
2013\end{array}$ & LFOB & CAF \\
\hline \multicolumn{8}{|l|}{$\begin{array}{l}\text { Antineoplásicos e agentes } \\
\text { imunomoduladores (ATC - L) }\end{array}$} \\
\hline Azatioprina & L04AX01 & $50 \mathrm{mg}$ & Comprimido & $\mathrm{S}$ & $\mathrm{S}$ & $\mathrm{S}$ & CEAF \\
\hline Subtotal & 1 & 1 & & 1 & 1 & 1 & 1 \\
\hline \multicolumn{8}{|l|}{ Sistema muscular esquelético (ATC - M) } \\
\hline Ibuprofeno & M01AE01 & $300 \mathrm{mg}$ & Comprimido & $\mathrm{S}$ & $\mathrm{N}$ & $\mathrm{S}$ & CBAF \\
\hline Alopurinol & M04AA01 & $100 \mathrm{mg}$ & Comprimido & $\mathrm{S}$ & $\mathrm{S}$ & $\mathrm{S}$ & CBAF \\
\hline Alendronato de sódio & M05BA04 & $70 \mathrm{mg}$ & Comprimido & $\mathrm{S}$ & $\mathrm{N}$ & $N$ & CBAF \\
\hline Subtotal & 3 & 3 & & 3 & 1 & 2 & 3 \\
\hline \multicolumn{8}{|l|}{ Sistema nervoso (ATC - N) } \\
\hline Ácido acetilsalicílico & N02BA01 & $500 \mathrm{mg}$ & Comprimido & $\mathrm{S}$ & $\mathrm{S}$ & $\mathrm{S}$ & CBAF \\
\hline Dipirona & N02BB02 & $500 \mathrm{mg} / \mathrm{mL}$ & Solução oral (gotas) & $\mathrm{N}$ & $\mathrm{N}$ & $\mathrm{S}$ & CBAF \\
\hline \multirow[t]{5}{*}{ Paracetamol } & N02BE01 & $500 \mathrm{mg}$ & Comprimido & $\mathrm{S}$ & $\mathrm{S}$ & $\mathrm{S}$ & CBAF/ \\
\hline & & & & & & & CESAF \\
\hline & & $200 \mathrm{mg} / \mathrm{mL}$ & Solução oral (gotas) & $\mathrm{S}$ & $\mathrm{N}$ & $\mathrm{S}$ & CBAF/ \\
\hline & & & & & & & CESAF \\
\hline & & $100 \mathrm{mg} / \mathrm{mL}$ & & $\mathrm{N}$ & $\mathrm{N}$ & $\mathrm{S}$ & NA \\
\hline Fenobarbital & N03AA02 & $100 \mathrm{mg}$ & Comprimido & $\mathrm{S}$ & $\mathrm{S}$ & $\mathrm{S}$ & CBAF \\
\hline Fenitoína & N03AB02 & $100 \mathrm{mg}$ & Comprimido & $\mathrm{S}$ & $\mathrm{S}$ & $\mathrm{S}$ & CBAF \\
\hline Clonazepam & N03AE01 & $2 \mathrm{mg}$ & Comprimido & $\mathrm{N}$ & $\mathrm{N}$ & $\mathrm{N}$ & NA \\
\hline Carbamazepina & N03AF01 & $200 \mathrm{mg}$ & Comprimido & $\mathrm{S}$ & $\mathrm{S}$ & $\mathrm{S}$ & CBAF \\
\hline Valproato de sódio & N03AG01 & $50 \mathrm{mg} / \mathrm{mL}$ & Xarope & $\mathrm{S}$ & $\mathrm{N}$ & $\mathrm{N}$ & CBAF \\
\hline Biperideno & N04AA02 & $2 \mathrm{mg}$ & Comprimido & $\mathrm{S}$ & $\mathrm{S}$ & $\mathrm{N}$ & CBAF \\
\hline Carbidopa + levodopa & N04BA02 & $25 m g+250 m g$ & Comprimido & $\mathrm{S}$ & $\mathrm{S}$ & $\mathrm{N}$ & CBAF \\
\hline Cloridrato de benserazida + levodopa & N04BA02 & $25 m g+100 m g$ & Comprimido & $\mathrm{S}$ & $\mathrm{N}$ & $\mathrm{N}$ & CBAF \\
\hline \multirow[t]{2}{*}{ Clorpromazina } & N05AA01 & $25 \mathrm{mg}$ & Comprimido & $\mathrm{S}$ & $\mathrm{S}$ & $\mathrm{S}$ & CBAF \\
\hline & & $100 \mathrm{mg}$ & Comprimido & $\mathrm{S}$ & $\mathrm{S}$ & $\mathrm{S}$ & CBAF \\
\hline \multirow[t]{3}{*}{ Haloperidol } & N05AD01 & $1 \mathrm{mg}$ & Comprimido & $\mathrm{S}$ & $\mathrm{N}$ & $\mathrm{S}$ & CBAF \\
\hline & & $5 \mathrm{mg}$ & & $\mathrm{S}$ & $\mathrm{S}$ & $\mathrm{S}$ & CBAF \\
\hline & & $2 \mathrm{mg} / \mathrm{mL}$ & Solução oral (gotas) & $\mathrm{S}$ & $\mathrm{S}$ & $\mathrm{N}$ & CBAF \\
\hline \multirow[t]{2}{*}{ Diazepam } & N05BA01 & $5 \mathrm{mg}$ & Comprimido sulcado & $\mathrm{S}$ & $\mathrm{S}$ & $\mathrm{S}$ & CBAF \\
\hline & & $10 \mathrm{mg}$ & & $\mathrm{N}$ & $\mathrm{S}$ & $\mathrm{S}$ & CBAF \\
\hline Cloridrato de amitriptilina & N06AA09 & $25 \mathrm{mg}$ & Comprimido & $\mathrm{S}$ & $\mathrm{S}$ & $\mathrm{S}$ & CBAF \\
\hline Fluoxetina & N06AB03 & $20 \mathrm{mg}$ & Comprimido & $\mathrm{S}$ & $\mathrm{S}$ & $\mathrm{S}$ & CBAF \\
\hline Subtotal & 15 & 22 & & 18 & 15 & 16 & 20 \\
\hline \multicolumn{8}{|l|}{ Antiparasitário, inseticida e repelentes } \\
\hline \multicolumn{8}{|l|}{$($ ATC - P) } \\
\hline Metronidazol & P01AB01 & $250 \mathrm{mg}$ & Comprimido & $\mathrm{S}$ & $\mathrm{S}$ & $\mathrm{S}$ & CBAF \\
\hline Benzoilmetronidazol & P01AB01 & $40 \mathrm{mg} / \mathrm{mL}$ & Suspensão oral & $\mathrm{S}$ & $\mathrm{S}$ & $\mathrm{S}$ & CBAF \\
\hline \multirow[t]{2}{*}{ Mebendazol } & P02CA01 & $100 \mathrm{mg}$ & Comprimido & $\mathrm{N}$ & $\mathrm{S}$ & $\mathrm{S}$ & NA \\
\hline & & $20 \mathrm{mg} / \mathrm{mL}$ & Suspensão oral & $\mathrm{N}$ & $\mathrm{N}$ & $\mathrm{S}$ & NA \\
\hline Albendazol & P02CA03 & $400 \mathrm{mg}$ & Comprimido mastigável & $\mathrm{S}$ & $\mathrm{S}$ & $\mathrm{N}$ & CBAF \\
\hline Benzoato de benzila & P03AX01 & $200 \mathrm{mg} / \mathrm{mL}$ & Emulsão & $\mathrm{N}$ & $\mathrm{N}$ & $\mathrm{N}$ & NA \\
\hline Subtotal & 4 & 6 & & 3 & 4 & 4 & 3 \\
\hline
\end{tabular}

(continua) 
Tabela 3 (continuação)

\begin{tabular}{|c|c|c|c|c|c|c|c|}
\hline Medicamento & ATC & Dose/Concentração & $\begin{array}{c}\text { Forma } \\
\text { farmacêutica }\end{array}$ & $\begin{array}{l}\text { RENAME } \\
2010\end{array}$ & $\begin{array}{l}\text { EML } \\
2013\end{array}$ & LFOB & CAF \\
\hline \multicolumn{8}{|l|}{ Sistema respiratório (ATC - R) } \\
\hline Dipropionato de beclometasona & R01AD01 & $50 \mathrm{mcg} /$ dose & $\begin{array}{c}\text { Administração tópica } \\
\text { nasal doseada }\end{array}$ & $\mathrm{S}$ & $\mathrm{N}$ & $\mathrm{N}$ & CBAF \\
\hline \multirow[t]{2}{*}{ Budesonida } & R01AD05 & $50 \mathrm{mcg} /$ dose & Administração tópica & $\mathrm{N}$ & $\mathrm{N}$ & $\mathrm{N}$ & CBAF \\
\hline & & $32 \mathrm{mcg} /$ dose & nasal doseada & $\mathrm{S}$ & $\mathrm{N}$ & $\mathrm{N}$ & CBAF \\
\hline Cloreto de sódio & R01AS & $9 \mathrm{mg} / \mathrm{mL}(0,9 \%)$ & Solução nasal & $\mathrm{S}$ & $\mathrm{N}$ & $\mathrm{S}$ & CBAF \\
\hline \multirow[t]{2}{*}{ Sulfato de salbutamol * } & $\mathrm{R03AC02}$ & $5 \mathrm{mg} / \mathrm{mL}$ & $\begin{array}{c}\text { Solução inalação - } \\
\text { administração pulmonar }\end{array}$ & $\mathrm{S}$ & $\mathrm{S}$ & $\mathrm{N}$ & CBAF \\
\hline & & $100 \mathrm{mcg} /$ dose & $\begin{array}{c}\text { Inalador doseado - } \\
\text { administração pulmonar }\end{array}$ & $\mathrm{S}$ & $\mathrm{S}$ & $\mathrm{N}$ & $\begin{array}{l}\text { CBAF/ } \\
\text { CEAF }\end{array}$ \\
\hline \multirow[t]{4}{*}{ Dipropionato de beclometasona * } & R03BA01 & $50 \mathrm{mcg} /$ dose & $\begin{array}{c}\text { Inalador doseado - } \\
\text { administração pulmonar }\end{array}$ & $\mathrm{S}$ & $\mathrm{S}$ & $\mathrm{N}$ & CBAF \\
\hline & & 200mcg/cápsula & $\begin{array}{l}\text { Cápsulas inalantes - } \\
\text { administração pulmonar }\end{array}$ & $\mathrm{S}$ & $\mathrm{N}$ & $\mathrm{N}$ & $\begin{array}{l}\text { CBAF/ } \\
\text { CEAF }\end{array}$ \\
\hline & & $200 \mathrm{mcg} /$ dose & $\begin{array}{c}\text { Inalador doseado - } \\
\text { administração pulmonar }\end{array}$ & $\mathrm{S}$ & $N$ & $\mathrm{~N}$ & $\begin{array}{l}\text { CBAF/ } \\
\text { CEAF }\end{array}$ \\
\hline & & $250 \mathrm{mcg} /$ dose & & $\mathrm{S}$ & $\mathrm{N}$ & $\mathrm{N}$ & $\begin{array}{l}\text { CBAF/ } \\
\text { CEAF }\end{array}$ \\
\hline \multirow[t]{2}{*}{ Brometo de ipratrópio * } & R03BB01 & $0,25 \mathrm{mg} / \mathrm{mL}$ & $\begin{array}{c}\text { Solução inalação - } \\
\text { administração pulmonar }\end{array}$ & $\mathrm{S}$ & $\mathrm{N}$ & $\mathrm{N}$ & CBAF \\
\hline & & $0,02 \mathrm{mg} /$ dose & $\begin{array}{c}\text { Inalador doseado - } \\
\text { administração pulmonar }\end{array}$ & $\mathrm{S}$ & $\mathrm{S}$ & $\mathrm{N}$ & CBAF \\
\hline \multirow[t]{2}{*}{ Sulfato de salbutamol * } & $\mathrm{R03CCO2}$ & $2 \mathrm{mg}$ & Comprimido sulcado & $\mathrm{N}$ & $\mathrm{N}$ & $\mathrm{S}$ & NA \\
\hline & & $0,4 \mathrm{mg} / \mathrm{mL}$ & Xarope & $\mathrm{N}$ & $\mathrm{N}$ & $\mathrm{S}$ & NA \\
\hline \multirow[t]{2}{*}{ Maleato de dexclorfeniramina } & $\mathrm{R} 06 \mathrm{AB} 02$ & $2 \mathrm{mg}$ & Comprimido & $\mathrm{S}$ & $\mathrm{N}$ & $\mathrm{N}$ & CBAF \\
\hline & & $0,4 \mathrm{mg} / \mathrm{mL}$ & Solução oral & $\mathrm{S}$ & $\mathrm{N}$ & $\mathrm{S}$ & CBAF \\
\hline Cloridrato de prometazina & R06AD02 & $25 \mathrm{mg}$ & Comprimido & $\mathrm{N}$ & $\mathrm{N}$ & $\mathrm{S}$ & CBAF \\
\hline Loratadina & R06AS13 & $10 \mathrm{mg}$ & Comprimido & $\mathrm{S}$ & $\mathrm{N}$ & $\mathrm{S}$ & CBAF \\
\hline Subtotal & 10 & 18 & & 14 & 4 & 6 & 16 \\
\hline \multicolumn{8}{|l|}{ Sistema ocular (ATC - S) } \\
\hline \multirow[t]{2}{*}{ Maleato de timolol } & S01ED01 & $0,25 \%$ & Solução oftalmológica & $\mathrm{S}$ & $\mathrm{S}$ & $\mathrm{N}$ & CBAF \\
\hline & & $0,50 \%$ & & $\mathrm{~S}$ & $\mathrm{~S}$ & $\mathrm{~N}$ & CBAF \\
\hline Subtotal & 1 & 2 & & 2 & 2 & 0 & 2 \\
\hline Total & 87 & 119 & & 96 & 63 & 71 & 102 \\
\hline
\end{tabular}

ATC: Anatomical Therapeutic Chemical; CAF: componente da assistência farmacêutica; CBAF: componente básico da assistência farmacêutica; CEAF: componente especializado da assistência farmacêutica; CESAF: componente estratégico da assistência farmacêutica; EML: Model List of Essential Medicines; LFOB: Laboratórios Farmacêuticos Oficiais do Brasil; N: não; NA: não se aplica; PFPB: Programa Farmácia Popular do Brasil; RENAME: Relação Nacional de Medicamentos Essenciais; S: sim.

* Medicamentos pertencentes ao rol daqueles totalmente subsidiados. 
Para exemplificar o problema, de acordo com os protocolos clínicos e diretrizes terapêuticas brasileiras 26 , sulfassalazina é um anti-inflamatório de segunda escolha, destinado ao tratamento de artrite reativa ou doença de Reiter. O paciente, antes de iniciar o tratamento com sulfassalazina, deve assinar um termo de esclarecimento e responsabilidade devido à possibilidade de desenvolver anemia aplásica, anemia hemolítica, plaquetopenia e/ou pneumonite intersticial. Para a dispensação de tal medicamento, nas farmácias do SUS que dispensam medicamentos do componente especializado, são exigidos exames laboratoriais (hemograma, urina tipo 1 e velocidade de sedimentação globular) que monitoram o aparecimento de reações adversas a medicamentos.

Azatioprina, imunossupressor utilizado como segunda escolha no tratamento de uveítes posteriores não infecciosas, na miastenia gravis e na dermatomiosite ou polimiosite, também necessita do termo de esclarecimento e responsabilidade assinado pelo paciente e de exames laboratoriais quinzenais até o terceiro mês de tratamento (hemograma, contagem de plaquetas e testes de função hepática) como exigência para o fornecimento do medicamento.

Doxiciclina é um antimicrobiano de uso sistêmico utilizado no tratamento de casos graves da malária, por Plasmodium falciparum, em combinação com quinina 27; na prevenção de malária 27 e no cólera 28. Essas doenças são de notificação compulsória 29 e o medicamento não poderia ser vendido para outros agravos à saúde devido ao risco de resistência microbiana, uso indevido e subnotificação, gerando outros problemas de saúde pública.

O principal enfoque do PFPB está relacionado à disponibilidade do produto farmacêutico que é uma das dimensões do acesso a medicamentos. As dimensões do acesso vão além de aspectos relacionados à efetividade, segurança $\mathrm{e}$ disponibilidade do medicamento, isto é, implica a atenção e o cuidado do paciente, usuário do sistema de saúde, como um todo 30,31 .
Esta pesquisa não pretende esgotar temas relacionados ao acesso ou mesmo à viabilidade do programa, mas indica limitações que podem ser superadas, considerando principalmente a política nacional de assistência farmacêutica vigente.

Em realidade, esta pesquisa faz uma análise mais profunda até o momento do elenco de medicamentos de um programa que aumentou, no Brasil, o acesso àqueles destinados à atenção básica e aos essenciais 32 por meio de copagamento entre o usuário e o Estado, com subsídios do Governo Federal na ordem de 356 milhões de Reais, em 2009 12, em um país no qual 77\% dos 191 milhões de habitantes (Agência Nacional de Saúde Suplementar. TabNet - Informações em saúde suplementar. http://www.ans.gov.br/anstabnet/ cgi-bin/tabnet?dados/tabnet_br.def\#, acessado em 06/Dez/2014; Instituto Brasileiro de Geografia e Estatística. XII Censo demográfico, 2010. http://www.censo2010.ibge.gov.br/, acessado em 21/Nov/13) utilizam o sistema público de saúde. Atualmente, é um programa com grande visibilidade internacional, sendo utilizado pela OMS como um estudo de caso bem-sucedido, que melhora o acesso e o uso de medicamentos 33 .

Considerando-se a importância que o PFPB vem adquirindo na política de saúde brasileira 13 e internacional 33 é necessário avaliá-lo regularmente. É preciso garantir a atualização do elenco com seleção de medicamentos minuciosa, tendo como base os critérios de essencialidade (de acordo com o conceito da OMS), os componentes da assistência farmacêutica no SUS, a produção por LFOB e a destinação à atenção básica, porta de entrada para qualquer sistema de saúde.

$\mathrm{O}$ alinhamento consistente do elenco de medicamentos do PFPB com as políticas de assistência farmacêutica no SUS poderia melhorar a qualidade da atenção à saúde, a gestão, a capacitação dos prescritores e a informação aos cidadãos. 


\section{Resumen}

El objetivo de este estudio fue analizar la lista de medicamentos del Programa Farmacia Popular de Brasil $(P F P B)$, en contraste con la política de la asistencia farmacéutica, en vigor en el país. El catálogo de medicamentos del PFPB se comparó con las listas de referencias de medicamentos esenciales (nacionales e internacionales), así como con la lista de financiación de los componentes de la asistencia farmacéutica del sistema de salud pública, y la producción pública de medicamentos por Laboratorios Oficiales de Farmacéuticos de Brasil (LOFB). El PFPB contiene 119 medicamentos de los que un 19,3\% y un 47,1\% no fueron seleccionados en las listas de referencias nacionales e internacionales, respectivamente; un 16,8\% no se utilizan en la atención primaria y un 40,3\% no son producidos por los LOFB. La revisión del catálogo de PFPB, basado en la concepción de medicamentos esenciales de la Organización Mundial de la Salud, el alineamiento político de la asistencia farmacéutica, y la producción por los LOFB es fundamental para mejorar la calidad de la asistencia de salud, la gestión, la formación de los prescriptores y la información a los ciudadanos.

Medicamentos Esenciales; Servicios Farmacéuticos; Programas Nacionales de Salud; Atención

Primaria de Salud

\section{Colaboradores}

S. M. Yamauti participou da idealização do artigo, trabalhou na análise e na interpretação dos dados, redigiu a primeira versão do artigo, aprovou a versão final a ser publicada e participou da sua revisão crítica. S. Barberato-Filho colaborou na idealização do artigo, auxiliou na redação, na sua revisão crítica e aprovou a versão final a ser publicada. L. C. Lopes participou da idealização do artigo, contribuiu na análise e interpretação dos dados, na revisão crítica e aprovou a versão final a ser publicada.

\section{Referências}

1. United Nations Human Rights. Universal declaration of human rights. http://www.ohchr.org/ EN/UDHR/Pages/UDHRIndex.aspx (acessado em 21/Nov/13).

2. Departamento de Atenção Básica, Secretaria de Políticas de Saúde, Ministério da Saúde. Política Nacional de Medicamentos. Brasília: Ministério da Saúde; 2001.

3. Câmara dos Deputados. Relatório da CPI-Medicamentos. http://www.crf-mt.org.br/arqs/mate ria/1362_a.pdf (acessado em 21/Nov/13).
4. Ministério da Saúde. Portaria GM/MS no 204, de 29 de janeiro de 2007. Regulamenta o financiamento e a transferência dos recursos federais para as ações e os serviços de saúde, na forma de blocos de financiamento, com o respectivo monitoramento e controle. Diário Oficial da União 2009; 31 jan.

5. Ministério da Saúde. Portaria GM/MS no 3.439, de 11 de novembro de 2010. Altera os Arts. 3o, 15, 16 e 63 e os Anexos I, II, III, IV e V à Portaria no 2.981/ GM/MS, de 26 de novembro de 2009, republicada em 1o de dezembro de 2009. Diário Oficial da União 2010; 17 nov. 
6. Ministério da Saúde. Portaria GM/MS no 2.981, de 26 de novembro de 2009. Aprova o Componente Especializado da Assistência Farmacêutica. Diário Oficial da União 2009, $1 \mathrm{dez}$.

7. Ministério da Saúde; Fundação Oswaldo Cruz. Programa Farmácia Popular do Brasil: manual básico. Brasília: Editora MS; 2005. (Série A. Normas e Manuais Técnicos).

8. Ministério da Saúde. Portaria GM no 971, de 15 de maio de 2012. Dispõe sobre o Programa Farmácia Popular do Brasil. Diário Oficial da União 2012; 17 mai.

9. Brasil. Lei no 8.666, de 21 de junho de 1993. Regulamenta o art. 37, inciso XXI, da Constituição Federal, institui normas para licitações e contratos da Administração Pública e dá outras providências. Diário Oficial da União 1993; 22 jun.

10. Santos-Pinto CDB, Miranda ES, Emmerick ICM, Costa NR, Osorio-de-Castro CGS. Preços e disponibilidade de medicamentos no Programa Farmácia Popular do Brasil. Rev Saúde Pública 2010; 44:611-9.

11. Santos-Pinto CDB, Costa NR, Osorio-de-Castro CGS. Quem acessa o Programa Farmácia Popular do Brasil? Aspectos do fornecimento público de medicamentos. Ciênc Saúde Coletiva 2011; 16:2963-73.

12. Instituto Brasileiro de Geografia e Estatística. Conta-Satélite de Saúde Brasil 2007-2009: análise dos resultados. http://www.ibge.gov.br/home/estatistica/economia/economia_saude/css_2007_2009/ comentario.pdf (acessado em 21/Nov/13).

13. Machado CV, Baptista TWF, Nogueira CO. Políticas de saúde no Brasil nos anos 2000: a agenda federal de prioridades. Cad Saúde Pública 2011; 27:521-32.

14. Ministério da Saúde. Laboratórios públicos produtores de medicamentos, soros e vacinas. http://portal.saude.gov.br/portal/saude/Gestor/ visualizar_texto.cfm?idtxt=32478 (acessado em 21/Nov/13).

15. Conselho Nacional de Saúde. Resolução no 338, de 06 de maio de 2004. Aprova a Política Nacional de Assistência Farmacêutica. Diário Oficial da União 2004; 20 mai.

16. Ministério da Saúde. Resolução RDC/ANVISA no 211, de 17 de novembro de 2006. Altera o Art. 1o da Resolução RDC no 111, de 29 de abril de 2005. Diário Oficial da União 2006; 20 nov.

17. WHO Collaborating Centre for Drug Statistics Methodology. Guidelines for ATC classification and DDD assignment 2013. Oslo: WHO Collaborating Centre for Drug Statistics Methodology; 2012.

18. WHO Expert Committee on the Selection and Use of Essential Medicines. The selection and use of essential medicines: report of the WHO Expert Committee, 2005 (including the 14th model list of essential medicines). Geneva: World Health Organization; 2006.
19. Departamento de Assistência Farmacêutica e Insumos Estratégicos, Secretaria de Ciência, Tecnologia e Insumos Estratégicos, Ministério da Saúde. Relação nacional de medicamentos essenciais: RENAME 2010. Brasília: Ministério da Saúde; 2010.

20. World Health Organization. WHO model list of essential medicines -18th list. http://www.who.int/ medicines/publications/essentialmedicines/en/index.html (acessado em 03/Jul/2013).

21. Ministério da Saúde. Portaria GM/MS no 533, de 28 de março de 2012. Estabelece o elenco de medicamentos e insumos da Relação Nacional de Medicamentos Essenciais (RENAME) no âmbito do Sistema Único de Saúde (SUS). Diário Oficial da União 2012; 29 mar.

22. Ministério da Saúde; Comissão Intergestores Tripartite. Resolução no 1, de 17 de janeiro de 2012. Estabelece as diretrizes nacionais da Relação Nacional de Medicamentos Essenciais (RENAME) no âmbito do Sistema Único de Saúde (SUS). Diário Oficial da União 2012; 18 jan.

23. Rodrigues MS. Produção pública de medicamentos no Brasil: uma análise sob a ótica da assistência farmacêutica no Sistema Único de Saúde [Dissertação de Mestrado]. Sorocaba: Programa de Pós-graduação em Ciências Farmacêuticas, Universidade de Sorocaba; 2012.

24. Pinto CDBS. O Programa Farmácia Popular do Brasil: modelo, cobertura e utilização frente à Política Nacional de Medicamentos [Dissertação de Mestrado]. Rio de Janeiro: Escola Nacional de Saúde Pública Sergio Arouca, Fundação Oswaldo Cruz; 2008.

25. Magalhães JL, Antunes AMS, Boechat N. Laboratórios farmacêuticos oficiais e sua relevância para saúde pública do Brasil. RECIIS Revista Eletrônica de Comunicação, Informação \& Inovação em Saúde 2011; 5:85-99.

26. Secretaria de Atenção à Saúde, Ministério da Saúde. Protocolos clínicos e diretrizes terapêuticas. Brasília: Ministério da Saúde; 2010.

27. World Health Organization. The selection and use of essential medicines: report of the WHO expert committee, march 2011 (including the 17th WHO model list of essential medicines and the 3rd WHO model list of essential medicines for children). Geneva: World Health Organization; 2012. (WHO Technical Report Series, 965).

28. Departamento de Assistência Farmacêutica e Insumos Estratégicos, Secretaria de Ciência, Tecnologia e Insumos Estratégicos, Ministério da Saúde. Formulário terapêutico nacional 2010: RENAME 2010. Brasília: Ministério da Saúde; 2010. 
29. Ministério da Saúde. Portaria no 104, de 25 de janeiro de 2011. Define as terminologias adotadas em legislação nacional, conforme o disposto no Regulamento Sanitário Internacional 2005 (RSI 2005), a relação de doenças, agravos e eventos em saúde pública de notificação compulsória em todo o território nacional e estabelece o fluxo, critérios, responsabilidades e atribuições aos profissionais e serviços de saúde. Diário Oficial da União 2011; 26 jan.

30. Hunt P, Khosla R. Acesso a medicamentos como um direito humano. Sur Rev Int Direitos Human 2008; 5:100-21.

31. Santos-Pinto CDB, Ventura M, Pepe VLE, Osoriode-Castro CGS. Novos delineamentos da Assistência Farmacêutica frente à regulamentação da Lei Orgânica da Saúde. Cad Saúde Pública 2013; 29:1056-8.
32. Secretaria de Ciência, Tecnologia e Insumos Estratégicos, Ministério da Sáude; Universidade Federal do Rio Grande do Sul. Pesquisa Nacional sobre Acesso, Utilização e Promoção do Uso Racional de Medicamentos no Brasil (PNAUM): pesquisa, informação e mais saúde para o Brasil. Primeiros resultados. http://www.ufrgs.br/pnaum/arqui vos/folders/primeiros-resultados (acessado em 06/ Dez/2014).

33. World Health Organization. The pursuit of responsible use of medicines: sharing and learning from country experiences. Technical report prepared for the Ministers Summit on the benefits of responsible use of medicines: setting policies for better and cost-effective health care. http:// apps.who.int/iris/bitstream/10665/75828/1/WHO EMP_MAR_2012.3_eng.pdf?ua=1 (acessado em 21/ Nov/2013).

Recebido em 05/Abr/2014

Versão final reapresentada em 13/Jan/2015

Aprovado em 05/Fev/2015 